Volume 70, Number 1, Pages 180-193(2021)

DOI: $10.31801 /$ cfsuasmas. 685733

ISSN 1303-5991 E-ISSN 2618-6470

Received by the editors: February 06, 2020; Accepted: December 05, 2020

\title{
CHANGE-CONSTRAINED STOCHASTIC PROGRAMMING PROBLEM WITH NORMAL, T AND SKEW NORMAL, SKEW T DISTRIBUTIONS
}

\author{
Gültaç EROĞLU İNAN \\ Department of Statistics, Faculty of Science, Ankara University, Ankara, TURKEY.
}

\begin{abstract}
In this paper, a change constrained optimization programming problem is studied under the assumption that the model coefficients in the inequalities defined as random variables are independent and assumed to be Normal, $t$; Non Normal Skew distributions; Skew Normal and Skew t distributions. The Hulkursar method transform the stochastic programming problem into a non-linear deterministic problem is used in the study. The most common distribution in CCSP is the Normal Distribution; but the real world problems always may not include normality. Therefore; in the practice stage, an application that the $a_{i j}$ technologic coefficient and the $b_{i}$ right side values in the inequalities have both Normal, $t$; Skew Normal and Skew $t$ distributions is given. Finally the obtained results have been compared.
\end{abstract}

\section{INTRODUCTION}

Practically, when the initial data is insufficient in a simple problem, making decisions with the data obtained is a rule. In the classical linear programming problem, model coefficients are defined as deterministic values. But many real life problems which are modelled as linear programming problems where coefficients appear as random variables. In the similar complex cases like that; the initial data is not known exactly (data uncertainty), there is a lack of data choosing deterministic models instead of stochastic models is more suitable. In this case, such problems are called as stochastic programming problem. The classical linear

2020 Mathematics Subject Classification. Primary 05C38, 15A15; Secondary 05A15, 15A18.

Keywords and phrases. Change constrained stochastic programming, skewness, skew normal distribution, skew t distribution.

geroglu@ankara.edu.tr

(D) ORCID ID: 0000-0002-3099-9949. 
programming problem is formulated as;

$$
\begin{aligned}
& \max z(x)=\sum_{j=1}^{n} c_{j} x_{j} \\
& \sum_{j=1}^{n} a_{i j} x_{j} \leq b_{i} \quad i=1, \ldots, m \\
& x_{j} \geq 0, j=1, \ldots, n
\end{aligned}
$$

where;

$a_{i j}$ : Technologic coefficient

$b_{i}$ : Right side value

$c_{j}$ : Objective function coefficient

$i=1, \ldots, m, j=1, \ldots, n$

In the classical problem all coefficients are deterministic. In the Stochastic Programming model, some or all model coefficients are described by random variables have known distributions rather than deterministic. Generally these distributions are assumed to be normal. There are many studies about stochastic programming in the literature. For a comprehensive survey of stochastic programming the reader is directed to I.M.Stancu [12].The stochastic programming problem should be transformed into a deterministic problem and then the basic deterministic model can be solved easily with the known methods. Change Constrained Programming (CCSP) and the Two Stage Programming are the most used methods for the model transformation. CCSP has first modelled by Charnes and Cooper 8. They have developed a new temporary planning of optimal stochastic decision rule under uncertainty. In the change constrained model, each constraints are defined by the certain probability. For a comprehensive survey of change constraint stochastic programming the reader is directed to Atalay [6, Yılmaz [15] are handled the Chi-Square type change constraints. Yllmaz 16 are handled the Gamma type chance constraints. In Atalay [6], the technologic coefficients $a_{i j}$ are assumed to be independent with Gamma Distribution. The deterministic constraints are obtained with the Essen Inequality. In the numerical experiments, Gamma and Normal Distribution approaches are compared. Yılmaz [17] are handled three methods are used to transform the change constraints into their equivalent deterministic constraints when the technologic coefficients are exponential random variables. These methods are based on the Central Limit Theorem. The fourth and the fifth methods are proposed for the deterministic equivalent of chance constraint. In the fourth method the exact distribution is used. In the last method, the exponential variates are transformed into the chi-squared variates. In an example, the five methods are compared to each other. Gamma, Exponential and Chi-Square are always positive distributions. But; there may not always be positivity. The most common distribution in statistics is the Normal Distribution; but the real world problems always may not include normality. Therefore, in the practice, the non-normal Distributions are more common than the Normal Distributions. In this study, for the skewness and the negativity, Skew Normal Distribution and Skew $t$ Distribution are used. The degree of distortion of symmetry in the normal distribution is called skewness. The Skew Distributions are obtained by the method add to skewness parameters in the known distributions. 
The Skew distributions family was first presented in the literature by Azzalini [1, 2].In this paper, the most used skew distributions the Skew Normal Distribution and the Skew $t$ Distributions proposed by the related study are used. The SkewNormal distribution shows variation depending on the skewness parameter. For the negative values of the $\lambda$ skewness parameter, the distribution is skewed to the right for the positive values of the $\lambda$ skewness parameter, the distribution is skewed to the left. If the skewness parameter is zero, the distribution becomes to standard normal distribution. For the details of the Skew Normal Distribution, the reader is directed to Azzalini 4], Azzalini [7]. The Skew-Normal density has very short tails. The Skew-Normal may be unsuitable where the distributions of the data have heavy tails. In this case; The Skew t distribution may be more suitable with heavier tails. The distribution shows variation depending on the skewness parameter similar to Skew Normal Distribution. For the details of the Skew t Distribution, the reader is directed to Azzalini [4], Arslan [5]. The reason for using the SkewNormal distribution in this study is that; providing flexibility to the application with modelling the normal and the close to normal distributions. The reasons for using the Skew $t$ distribution in this study are that; it has Normal Distribution, Skew Normal Distribution, Student's- $t$ Distribution; it is a good alternative to normal distribution. This remainder of this paper is organized as follows: In Section 2, the change constrained stochastic programming is introduced. And then four cases where the model coefficients are defined as random variables are handled. For each cases; the deterministic equivalent of the stochastic model is given. In Section 3, Skew Normal and Skew $t$ distributions and statistical properties are introduced. In Section 4, two CCSP problems where the model coefficients are random variables have the Normal, Skew Normal and Skew t distributions are solved. This is the first study handle the CCSP where the model coefficients $a_{i j}, c_{j}, b i$ are given as random variables have the skewness distributions: Skew Normal and Skew $t$. The motivation of this paper comes from the fact that; the real World problems always not include normality. We try to compare the Normal, $t$ and non-normal Skew distributions for the CCSP. To the best of our knowledge this is the first study include the Skew Normal and Skew $t$ instead of Normal Distribution for CCSP. In the CCSP problems; in the literature it is usually assumed that the $a_{i j}, b i$ random variables have normal distribution. The problems are handled with the different $\mu, \sigma^{2}$ pameter values and their effects are investigated. To the best of our knowledge, there is no previous work assuming Skew Distributions as a constraint distribution in the context of CCSP. The Skew distributions are essential in CCSP because the normality assumptions may not always be provided in the Real World problems. The normal distributions symmetry property becomes problem and skewness is present and important issue in the real applications. The skewness and the tick tail effects can be investigated by the skew distributions in CCSP. The advantages of using the Skew Normal distribution in CCSP is that; they provides us flexibility for the random variables $a_{i j}$, bi have the normal distribution shape but with skewness. SN 
is considered as an the alternative of the normal distribution have different values of skewness and kurtosis. The advantages of using these Skew t distribution in CCSP is that; the distribution include the alternative distributions of normal distribution with the skewness and the heavier tails. In practice Skew Distributions are very useful distributions because of their properties. Therefore; in this study we want to show the usability of the Skew Distributions in the CCSP. We want to handle the CCSP with the $\mu, \sigma^{2}$ and the $\lambda$ skewness parameters.

\section{Change-Constrained Stochastic Programming}

The measured gene expression levels may be modeled as

$$
\begin{gathered}
\max (\min ) z(x)=\sum_{j=1}^{n} c_{j} x_{j} \\
P\left[\sum_{j=1}^{n} a_{i j} x_{j} \leq b_{i}\right] \geq 1-u_{i} \\
x_{j} \geq 0 \quad j=1, \ldots, n \\
u_{i} \epsilon(0,1), \quad i=1, \ldots, m
\end{gathered}
$$

where; $a_{i j}, c_{j}, b_{i} \quad$ random variables, $P\left[\sum_{j=1}^{n} a_{i j} x_{j} \leq b_{i}\right] \geq 1-u_{i}$ the $i$. th change constraint, $1-u_{i}$ : lower bound for the $i$. change constraint (specified probability), $x_{j}$ : decision variable $i=1, \ldots, m, j=1, \ldots, n$.

In the model it is assumed that; $a_{i j}, c_{j}, b_{i}$ are normally distributed random variables with known mean and variances, $x_{j}$ decision variables are deterministic. In Hulsurkar 11], There are seven case may be occurred are handled. These cases are given as;

Only $a_{i j}$, only $c_{j}$ or only $b_{i}$ are random variables (three cases).

Together $a_{i j}, b_{i}$ are random variables.

Together $a_{i j}, c_{j}$ are random variables.

Together $c_{j}, b_{i}$ are random variables.

Together $a_{i j}, c_{j}, b_{i}$ are random variables.

The deterministic equivalents of the probabilistic programming problems are given in the study for the fourth cases. The other ones can be easily obtained as similar.

Case 1 : Only $a_{i j}$ coefficients are random variables.

Let's the covariance between $a_{i j}$ and $a_{k l}$ random variables are known.

Random variable $d_{i}$ is given as;

$$
d_{i}=\sum_{j=1}^{n} a_{i j} x_{j}, \quad i=1, \ldots, m
$$

where, $a_{i 1}, \ldots, a_{i n}$ normal random variables and $x_{1}, \ldots, x_{n}$ decision variables. The expected values and the variance of $d_{i}$ random variable are obtained as blow;

$$
E\left(d_{i}\right)=\sum_{j=1}^{n} E\left(a_{i j} x_{j}\right), \mathrm{i}=1, \ldots, \mathrm{m}
$$




$$
\operatorname{Var}\left(d_{i}\right)=X^{T} V_{i} X, \mathrm{i}=1, \ldots, \mathrm{m}
$$

The deterministic non-linear equivalent of the probabilistic programming problem.

$$
\begin{gathered}
\sum_{j=1}^{n} E\left(a_{i j}\right) x_{j}+K_{u_{i}} \sqrt{X^{T} V_{i} X} \leq b_{i} \quad i=1, \ldots, m \\
x_{j} \geq 0 \quad j=1, \ldots, n
\end{gathered}
$$

where; $K_{u_{i}}$ is the value of standard normal variable and $\Phi\left(K_{u_{i}}\right): 1-u_{i}$. If the $a_{i j}$ random variables are independent the inequalities constraint $(6)$ can be written as:

$$
\sum_{j=1}^{n} E\left(a_{i j}\right) x_{j}+K_{u_{i}} \sqrt{\operatorname{Var}\left(a_{i j}\right) x_{j}^{2}} \leq b_{i} \quad i=1, \ldots, m
$$

Case 2: Only $b_{i}$ coefficients are random variables, the deterministic equivalent model of the stochastic model is given as;

$$
\begin{aligned}
& \max (\min ) z(x)=\sum_{j=1}^{n} c_{j} x_{j} \\
& \sum_{j=1}^{n} a_{i j} x_{j} \leq E\left(b_{i}\right)+K_{u_{i}} \sqrt{\operatorname{Var}\left(b_{i}\right)} \quad i=1, \ldots, m \\
& x_{j} \geq 0 \quad j=1, \ldots, n
\end{aligned}
$$

Case 3: Only $c_{j}$ coefficients are random variables, the deterministic equivalent model of the stochastic model is given as;

$$
\max (\min ) E(z(x))=\sum_{j=1}^{n} E\left(c_{j}\right) x_{j}
$$

Case 4: $a_{i j}$ and $b_{i}$ coefficients are together random variables, the deterministic equivalent model of the stochastic model is given as;

$$
\begin{gathered}
\max (\min )(z(x))=\sum_{j=1}^{n}\left(c_{j}\right) x_{j} \\
E\left(\sum_{j=1}^{n} a_{i j} x_{j}-b_{i}\right)+K_{u_{i}} \sqrt{\operatorname{Var}\left(\sum_{j=1}^{n} a_{i j} x_{j}-b_{i}\right)} \leq 0 \quad i=1, \ldots, m
\end{gathered}
$$

For the proofs the reader is directed to Hulsurkar 11.

\section{Skew Distributions}

3.1. Skew Normal Distribution. Normal Distribution is a preferred distribution through the analytic case in the studied, connection with central limit theory, collability and the other advantage properties. However, there are many situations in which the normal distribution assumption is not provided in practice. As it is a symmetrical distribution, the usage area of the normal distribution becomes limited. Therefore, the problem of deviation from symmetry in the normal distribution has been tried to be solved in some of these distributions. One such as distribution is skew normal distribution. 
While the normal distribution can only determine a shape and scale parameter, the skew normal distribution also has a shape parameter in addition to these parameters. This parameter is also called the skewness parameter. The parameter determines the skewness of the distribution. In practice this is a very useful distribution because of this property. Because skewness is present in almost in real data. However the skew normal distribution includes many features found in the normal distribution.

Skew-Normal distribution was first mentioned in the study O'Hagan and Leonhard [14. Then Azzalini [1,2] extended the theory of the skew normal distribution. Skew normal distribution include the normal distribution. At the same time; it is ease to operate with the skew normal distribution. For these reasons the distribution is widely used in the literature. If a random variable $Z$ has density function

$$
f(z ; \lambda)=2 f(z) F(\lambda z) \quad-\infty<z<\infty
$$

where; $f$ : the standard normal density and distribution function, $F$ : the standard normal distribution function, $\lambda$ : skewness parameter, $Z$ is a skew-normal random variable with parameter $\lambda$; it is denoted $Z \sim \operatorname{SN}(\lambda)$.

General Form of 11

$$
\begin{aligned}
& Y=\mu+\sigma Z \\
& f(y)=\frac{2}{\sigma} f\left(\frac{y-\mu}{\sigma}\right) F\left(\lambda\left(\frac{y-\mu}{\sigma}\right)\right)
\end{aligned}
$$

where: $\mu \in R$ : location parameter $\sigma \in R^{+}$: scale parameter. If the random variable $Y$ has $S N$ distribution with the parameters; $\mu, \sigma, \lambda$; it is denoted as $Y \sim$ $\mathrm{SN}(\mu, \sigma, \lambda)$. The expected value and the variance of the $Y$ is given as;

$$
\begin{aligned}
& E(Y)=\mu+\sqrt{\frac{2}{\pi}} \delta_{\lambda} \delta \\
& \operatorname{Var}(Y)=\left(1-\frac{2}{\pi} \delta^{2} \lambda\right) \sigma^{2}
\end{aligned}
$$

where;

$$
\delta_{\lambda}=\frac{\lambda}{\sqrt{1+\lambda^{2}}}
$$

The skewness and the kurtosis of the $Z \sim \mathrm{SN}(\lambda)$ are obtained as;

$$
\begin{aligned}
\gamma_{1} & =\frac{\sqrt{2}(4-\pi) \lambda^{3}}{\left(\pi+(\pi-2) \lambda^{2}\right)^{\frac{3}{2}}} \\
\gamma_{2} & =3+\frac{8(\pi-3) \lambda^{4}}{\left(\pi+(\pi-2) \lambda^{2}\right)^{2}}
\end{aligned}
$$

Lin et al. 13 The skewness and the kurtosis values of the $S N$ distributions for some $\lambda$ values was obtained as below; 
TABLE 1. The skewness and the kurtosis values of $S N(\lambda)$

\begin{tabular}{|c|l|l|l|l|l|}
\hline$\lambda$ & 0 & 0.1 & 0.5 & 0.9 & 1 \\
\hline$\gamma_{1}$ & 0 & 0.0002 & 0.0239 & 0.1079 & 0.1369 \\
\hline$\gamma_{2}$ & 3 & 3 & 3.0060 & 3.0449 & 3.0617 \\
\hline
\end{tabular}

3.2. Skew $t$ Distribution. Let $Y$ random variable has the Skew $t$ distribution with the $\mu$ location parameter, $\sigma^{2} \epsilon(0, \infty)$ scale parameter, skewness parameter and with $v \epsilon(0, \infty)$ degree of freedom improved by Gupta et al [9], Azzalini and Capitanio 3 and Gupta 10. Y random variable is defined as;

$$
Y=\frac{X}{\sqrt{\frac{V}{v}}}
$$

where; $X$, Skew Normal Distribution with parameter $\lambda V, \chi^{2}(v) Y$ and $V$ are independent each other's, $v$ : the degree of freedom. The probability density function of Skew $t$ distribution is defined as;

$$
f_{s t}(y ; \lambda)=2 t_{v}(x) T_{v+1}\left(\lambda x \sqrt{\frac{v+1}{x^{2}+v}}\right)
$$

where

$$
x=\frac{y-\mu}{\sigma}
$$

$t_{v}$ : The probability density function of $t$ distribution with $t$ degree of freedom. $T_{v+1}$ : The distribution function of $t$ distribution with $v+1$ degree of freedom. $\lambda$ : Skewness parameter.

If $Y$ random variable has the pdf (17) it is denoted as $Y \sim S t_{v}\left(\mu, \sigma^{2}, \lambda, v\right)$. The expected value and the variance of $Y \sim S t_{y}\left(\mu, \sigma^{2}, \lambda, v\right)$ are obtained as follows;

$$
\begin{gathered}
E(Y)=\mu+\left(\left(\sqrt{\frac{v}{\pi}} \frac{\lambda \Gamma\left(\frac{v-1}{2}\right)}{\sqrt{\lambda^{2}+1} \Gamma\left(\frac{v}{2}\right)}\right) \sigma\right), v>1 \\
V(Y)=\left(\frac{v}{v-2}-c^{2}\right) \sigma^{2}, \quad v>2 \\
c=\sqrt{\frac{v}{\pi}} \delta_{\lambda}\left(\frac{\Gamma\left(\frac{v-1}{2}\right)}{\Gamma\left(\frac{v}{2}\right)}\right) \\
\delta_{\lambda}=\frac{\lambda}{\sqrt{\lambda^{2}+1}}
\end{gathered}
$$

Azzalini and Capitanio 3.

The skewness and the kurtosis of the $S t$ are obtained as 


$$
\begin{aligned}
& \gamma_{1}=c\left(\frac{v\left(3-\delta_{\lambda}^{2}\right)}{v-3}-\frac{3 v}{v-2}+2 c^{2}\right)\left(\frac{v}{v-2}-c^{2}\right)^{-\frac{3}{2}} \quad v>3 \\
& \gamma_{2}=\frac{\left(\frac{3 v^{2}}{(v-2)(v-4)}-\frac{4 c^{2} v\left(3-\delta_{\lambda}^{2}\right)}{v-3}+\frac{6 c^{2} v}{v-2}-3 c^{4}\right)}{\left(\frac{v}{v-2}-c^{2}\right)^{2}}-3, \quad v>4
\end{aligned}
$$

where

$$
\delta_{\lambda}=\frac{\lambda}{\sqrt{1+\lambda^{2}}} \quad c=\delta_{\lambda}(v / \pi)^{\frac{1}{2}} \frac{\Gamma\left(\frac{v-1}{2}\right)}{r\left(\frac{v}{2}\right)}
$$

The skewness and the kurtosis values of the $S_{t}$ distributions for some $\lambda, v$ values were obtained in Table 2 .

TABLE 2. The skewness and the kurtosis values of the $S t_{v}(0,1, \lambda)$

\begin{tabular}{|c|c|c|c|c|c|}
\hline & $\lambda=0$ & $\lambda=0.1$ & $\lambda=0.5$ & $\lambda=0.9$ & $\lambda=1$ \\
\hline$v$ & $\gamma_{1} \gamma_{2}$ & $\gamma_{1} \gamma_{2}$ & $\gamma_{1} \gamma_{2}$ & $\gamma_{1} \gamma_{2}$ & $\gamma_{1} \gamma_{2}$ \\
\hline 3 & -- & -- & -- & -- & -- \\
\hline 5 & 09 & 0.11039 .0324 & 0.55279 .8020 & 0.977611 .4330 & 1.075811 .9208 \\
\hline 10 & 04 & 0.03334 .0017 & 0.18394 .0560 & 0.37364 .2285 & 0.42434 .2909 \\
\hline
\end{tabular}

\section{Numerical Example}

Let's define the CCSP model as follows;

$$
\begin{gathered}
\max Z=7 x_{1}+2 x_{2}+4 x_{3} \\
P\left(a_{11} x_{1}+a_{12} x_{2}+a_{13} x_{3} \leq 8\right) \geq 0.95 \\
P\left(5 x_{1}+x_{2}+6 x_{3} \leq b_{2}\right) \geq 0.10 \\
x_{1}, x_{2}, x_{3} \geq 0
\end{gathered}
$$

By using the inequality constraint (2.6), (2.7) in the Case 1 and Case 2 , the deterministic equality of the problem 24 is written as generally form.

$$
\begin{gathered}
\max Z=7 x_{1}+2 x_{2}+4 x_{3} \\
E\left(a_{11}\right) x_{1}+E\left(a_{12}\right) x_{2}+E\left(a_{12}\right) x_{2}+K_{0.95} x_{4}^{2} \leq 8 \\
5 x_{1}+x_{2}+6 x_{3} \leq E\left(b_{2}\right)+K_{0.10} \sqrt{\operatorname{Var}\left(b_{2}\right)} \\
\operatorname{Var}\left(a_{11}\right) x_{1}^{2}+\operatorname{Var}\left(a_{12}\right) x_{2}^{2}+\operatorname{Var}\left(a_{13}\right) x_{3}^{2}-x_{4}^{2}=0 \\
x_{1}, x_{2}, x_{3}, x_{4} \geq 0
\end{gathered}
$$

where, $K_{0,00}=1.285$ and $K_{0,95}=1.645$.

Now, we assume that $a_{i j}, j=1,2,3$ and $b_{2}$ are independent Normal Random Variables with the following randomly means and variances 


$$
\begin{array}{cl}
E\left(a_{11}\right)=4 & \operatorname{Var}\left(a_{11}\right)=4 \\
E\left(a_{12}\right)=4 & \operatorname{Var}\left(a_{12}\right)=8 \\
E\left(a_{13}\right)=6 & \operatorname{Var}\left(a_{13}\right)=12 \\
E\left(b_{2}\right)=7 & \operatorname{Var}\left(b_{2}\right)=9
\end{array}
$$

For these values, the problem 25 turns into the following deterministic problem

$$
\begin{gathered}
\max z=7 x_{1}+2 x_{2}+4 x_{3} \\
4 x_{1}+4 x_{2}+6 x_{3}+1.645 x_{4}^{2} \leq 8 \\
5 x_{1}+x_{2}+6 x_{3} \leq 10.855 \\
4 x_{1}^{2}+8 x_{2}^{2}+12 x_{3}^{2}-x_{4}^{2}=0 \\
x_{1}, x_{2}, x_{3}, x_{4} \geq 0
\end{gathered}
$$

By Lingo package program, the solution of the problem 26 is obtained as:

$$
X^{*}=\left[\begin{array}{c}
1.097394 \\
0 \\
0 \\
2.194787
\end{array}\right] \quad Z^{*}=7.681754
$$

Now we assume that; $a_{i j}, j=1,2,3$ and $b_{2}$ are independent Skew Normal Random Variables with the $\lambda$ skewness parameter.

TABLE 3 . The Expected values and the variances of the $a_{i j}$ random variables for each $\lambda$ values for Skew Normal Distribution.

\begin{tabular}{|c|c|c|c|c|c|}
\hline & $\lambda=0$ & $\lambda=0.1$ & $\lambda=0.5$ & $\lambda=0.9$ & $\lambda=1$ \\
\hline$E\left(a_{11}\right)$ & 4 & 4.1588 & 4.7136 & 5.0675 & 5.1284 \\
\hline$E\left(a_{12}\right)$ & 4 & 4.2246 & 5.0093 & 5.5097 & 5.5998 \\
\hline$E\left(a_{13}\right)$ & 6 & 6.2750 & 7.2361 & 7.8490 & 7.9544 \\
\hline $\operatorname{Var}\left(a_{11}\right)$ & 4 & 3.9748 & 3.4907 & 2.8604 & 2.7268 \\
\hline $\operatorname{Var}\left(a_{12}\right)$ & 8 & 7.9495 & 6.9813 & 5.7207 & 5.4534 \\
\hline $\operatorname{Var}\left(a_{13}\right)$ & 12 & 11.9244 & 10.4721 & 8.5812 & 8.1803 \\
\hline$E\left(b_{2}\right)$ & 7 & 7,2382 & 8,0705 & 8,6013 & 8,6926 \\
\hline $\operatorname{Var}\left(b_{2}\right)$ & 9 & 8,9433 & 7,8541 & 6.4359 & 6,1352 \\
\hline
\end{tabular}

In Table 3 and Table 4 ; for some values of $\lambda$ the expected values and the variances of the $a_{i j}, b_{i}$ random variables and the right side values of the constraint 2 of the problem 25 are given. It is assumed that the skewness parameter is known.

The optimal solutions of the problem 25 under the Skew Normal Distribution assumption for each skewness values are given in Table 5 .

In this step, we assume that; $a_{i j}, i=1, j=1,2,3$ and $b_{2}$ are independent Skew$t$ random variables with the $\lambda, v$ parameters. The expected values, variances of the random variables and the right hand values of the second constraint of the problem 25 are given in Table 6 and Table 7. The degree of freedom $v$ is known in the study. 
TABLE 4 . The expected values and the variances of the $b_{i}$ random variables and the right side values of the second constraint of the problem (25)

\begin{tabular}{|c|c|c|c|c|c|}
\hline & $\lambda=0$ & $\lambda=0.1$ & $\lambda=0.5$ & $\lambda=0.9$ & $\lambda=1$ \\
\hline$E\left(b_{2}\right)$ & 7 & 7.2382 & 8.0705 & 8.6013 & 8.6926 \\
\hline $\operatorname{Var}\left(b_{2}\right)$ & 9 & 8.9433 & 7.8541 & 6.4359 & 6.1352 \\
\hline Right side values of constraint 2 & 10.855 & 11.081 & 11.6717 & 11.8612 & 11.8755 \\
\hline
\end{tabular}

TABle 5. The optimal solution of the problem (25) for each $\lambda$ values

\begin{tabular}{|c|c|c|c|c|c|}
\hline & $\lambda=0$ & $\lambda=0.1$ & $\lambda=0.5$ & $\lambda=0.9$ & $\lambda=1$ \\
\hline$X_{1}$ & 1.0973 & 1.0754 & 1.0273 & 1.0191 & 1.0197 \\
\hline$X_{2}$ & 0 & 0 & 0 & 0 & 0 \\
\hline$X_{3}$ & 0 & 0 & 0 & 0 & 0 \\
\hline$X_{4}$ & 2.1947 & 2.1442 & 1.9194 & 1.7236 & 1.6839 \\
\hline Max $Z=$ & 7.6817 & 7.5284 & 7.1914 & 7.1348 & 7.1340 \\
\hline
\end{tabular}

TABLE 6. The Expected values and the variances of the $a_{i j}$ random variables for each $\lambda$ and $v$ values for the Skew-t distribution

\begin{tabular}{|c|c|c|c|c|c|}
\multicolumn{1}{|c|}{$v=3$} \\
\hline$E\left(a_{\mu}\right)$ & $\lambda=0$ & $\lambda=0.1$ & $\lambda=0.5$ & $\lambda=0.9$ & $\lambda=1$ \\
\hline$E\left(a_{12}\right)$ & 4 & 4.2194 & 4.9862 & 5.4753 & 5.5594 \\
\hline$E\left(a_{13}\right)$ & 6 & 6.3103 & 5.3948 & 6.0863 & 6.2053 \\
\hline $\operatorname{Var}\left(a_{11}\right)$ & 12 & 11.9518 & 11.0273 & 9.8236 & 9.5683 \\
\hline $\operatorname{Var}\left(a_{12}\right)$ & 23.9995 & 23.9032 & 22.0542 & 19.6467 & 19.1362 \\
\hline $\operatorname{Var}\left(a_{13}\right)$ & 36 & 35.8555 & 33.0819 & 29.4706 & 28.7048 \\
\hline
\end{tabular}

\begin{tabular}{|c|c|c|c|c|c|}
\hline & $\lambda=0$ & $\lambda=0.1$ & $\lambda=0.5$ & $\lambda=0.9$ & $\lambda=1$ \\
\hline$E\left(a_{\mu}\right)$ & 4 & 4.1889 & 4.8488 & 5.2697 & 5.3421 \\
\hline$E\left(a_{12}\right)$ & 4 & 4.2671 & 5.2004 & 5.7956 & 5.8980 \\
\hline$E\left(a_{13}\right)$ & 6 & 6.3271 & 7.4702 & 8.1992 & 8.3246 \\
\hline $\operatorname{Var}\left(a_{11}\right)$ & 6.6667 & 6.6310 & 5.9462 & 5.0545 & 4.8654 \\
\hline $\operatorname{Var}\left(a_{12}\right)$ & 13.3331 & 13.2617 & 11.8921 & 10.1088 & 9.7306 \\
\hline $\operatorname{Var}\left(a_{13}\right)$ & 20 & 19.8930 & 17.8385 & 15.1634 & 14.5962 \\
\hline
\end{tabular}

$$
v=10
$$




\begin{tabular}{|c|c|c|c|c|c|}
\hline & $\lambda=0$ & $\lambda=0.1$ & $\lambda=0.5$ & $\lambda=0.9$ & $\lambda=1$ \\
\hline$E\left(a_{11}\right)$ & 4 & 4.1721 & 4.7734 & 5.1569 & 5.2228 \\
\hline$E\left(a_{12}\right)$ & 4 & 4.2434 & 5.0937 & 5.6361 & 5.7294 \\
\hline$E\left(a_{12}\right)$ & 6 & 6.2980 & 7.3396 & 8.0038 & 8.1180 \\
\hline $\operatorname{Var}\left(a_{11}\right)$ & 5 & 4.9704 & 4.4019 & 3.6616 & 3.5046 \\
\hline $\operatorname{Var}\left(a_{12}\right)$ & 9.9998 & 9.9406 & 8.8035 & 7.3231 & 7.0091 \\
\hline $\operatorname{Var}\left(a_{13}\right)$ & 15 & 14.9112 & 13.2056 & 10.9848 & 10.5139 \\
\hline
\end{tabular}
$v=100$

\begin{tabular}{|c|c|c|c|c|c|}
\hline & $\lambda=0$ & $\lambda=0.1$ & $\lambda=0.5$ & $\lambda=0.9$ & $\lambda=1$ \\
\hline$E\left(a_{11}\right)$ & 4 & 4.1600 & 4.7191 & 5.0756 & 5.1369 \\
\hline$E\left(a_{12}\right)$ & 4 & 4.2263 & 5.0169 & 5.5211 & 5.6078 \\
\hline$E\left(a_{12}\right)$ & 6 & 6.2771 & 7.2454 & 7.8630 & 7.9692 \\
\hline $\operatorname{Var}\left(a_{11}\right)$ & 4.0816 & 4.0560 & 3.5646 & 2.9247 & 2.7890 \\
\hline $\operatorname{Var}\left(a_{12}\right)$ & 8.1631 & 8.1119 & 7.1290 & 5.8493 & 5.5779 \\
\hline $\operatorname{Var}\left(a_{13}\right)$ & 12.2449 & 12.1681 & 10.6938 & 8.7741 & 8.3671 \\
\hline
\end{tabular}

TABLE 7 . The expected values and the variances of the $b_{2}$ random variables and the right side values of the second constraint of the problem (25) for the Skew- $t$ distribution.

\begin{tabular}{|c|c|c|c|c|c|}
\hline & $\lambda=0$ & $\lambda=0.1$ & $\lambda=0.5$ & $\lambda=0.9$ & $\lambda=1$ \\
\hline$E\left(b_{2}\right)$ & 7 & 7.3292 & 8.4794 & 9.2129 & 9.3391 \\
\hline Var $\left(b_{2}\right)$ & 27 & 26.8917 & 24.8115 & 22.1030 & 21.5287 \\
\hline Right side values & 13.6771 & 13.9929 & 14.8801 & 15.2542 & 15.3014 \\
\hline \multicolumn{7}{|c|}{$v=5$} \\
\hline$E\left(b_{2}\right)$ & $\lambda=0$ & $\lambda=0.1$ & $\lambda=0.5$ & $\lambda=0.9$ & $\lambda=1$ \\
\hline $\operatorname{Var}\left(b_{2}\right)$ & 15 & 14.9197 & 13.3789 & 11.3726 & 10.9472 \\
\hline Right side values & 11.9768 & 12.2467 & 12.9734 & 13.238 & 13.2648 \\
\hline \multicolumn{5}{|c|}{$v=10$} \\
\hline$E\left(b_{2}\right)$ & 7 & 7.2581 & 8.1601 & 8.7353 & 8.8343 \\
\hline $\operatorname{Var}\left(b_{2}\right)$ & 11.25 & 11.1834 & 9.9042 & 8.2386 & 7.8854 \\
\hline Right side values & 13.31 & 11.5553 & 12.2041 & 12.4236 & 12.4427 \\
\hline \multicolumn{7}{|c|}{$v=100$} \\
\hline$E\left(b_{2}\right)$ & $\lambda=0$ & $\lambda=0.1$ & $\lambda=0.5$ & $\lambda=0.9$ & $\lambda=1$ \\
\hline $\operatorname{Var}\left(b_{2}\right)$ & 9.1837 & 9.1261 & 8.0203 & 6.5806 & 6.2753 \\
\hline Right side values & 10.8941 & 11.1219 & 11.7177 & 11.9098 & 11.9244 \\
\hline
\end{tabular}


The optimal solutions of the problem (25) under the Skew-t distribution assumption for each skewness values are given in Table 8 .

TABLE 8. The optimal solution of the problem (25) for each $v, \lambda$ values

\begin{tabular}{|c|c|c|c|c|c|}
\hline \multicolumn{6}{|c|}{$v=3$} \\
\hline & $\lambda=0$ & $\lambda=0.1$ & $\lambda=0.5$ & $\lambda=0.9$ & $\lambda=1$ \\
\hline$X_{1}$ & 0.8248 & 0.8075 & 0.7656 & 0.7525 & 0.7513 \\
\hline$X_{2}$ & 0 & 0 & 0 & 0 & 0 \\
\hline$X_{3}$ & 0 & 0 & 0 & 0 & 0 \\
\hline$X_{4}$ & 2.8574 & 2.7918 & 2.5424 & 2.3585 & 2.3240 \\
\hline $\operatorname{Max} Z=$ & 5.774 & 5.6529 & 5.3594 & 5.2675 & 5.2592 \\
\hline \multicolumn{6}{|c|}{$v=5$} \\
\hline & $\lambda=0$ & $\lambda=0.1$ & $\lambda=0.5$ & $\lambda=0.9$ & $\lambda=1$ \\
\hline$X_{1}$ & 0.9700 & 0.9495 & 0.9029 & 0.8920 & 0.8918 \\
\hline$X_{2}$ & 0 & 0 & 0 & 0 & 0 \\
\hline$X_{3}$ & 0 & 0 & 0 & 0 & 0 \\
\hline$X_{4}$ & 2.5045 & 2.4452 & 2.1017 & 2.0055 & 1.9671 \\
\hline $\operatorname{Max} Z=$ & 6.790 & 6.6469 & 6.3204 & 6.2444 & 6.2426 \\
\hline \multicolumn{6}{|c|}{$\begin{array}{c}v=10 \\
\lambda=01\end{array}$} \\
\hline & $\lambda=0$ & $\lambda=0.1$ & $\lambda=0.5$ & $\lambda=0.9$ & $\lambda=1$ \\
\hline$X_{1}$ & 1.0418 & 1.0204 & 0.9726 & 0.9633 & 0.9635 \\
\hline$X_{2}$ & 0 & 0 & 0 & 0 & 0 \\
\hline$X_{3}$ & 0 & 0 & 0 & 0 & 0 \\
\hline$X_{4}$ & 2.3297 & 2.2750 & 2.0407 & 1.8433 & 1.8038 \\
\hline $\operatorname{Max} Z=$ & 7.2932 & 7.1432 & 6.8087 & 6.7450 & 6.7432 \\
\hline \multicolumn{6}{|c|}{$v=100$} \\
\hline & $\lambda=0$ & $\lambda=0.1$ & $\lambda=0.5$ & $\lambda=0.9$ & $\lambda=1$ \\
\hline$X_{1}$ & 1.0923 & 1.0705 & 1.0223 & 1.0140 & 1.0147 \\
\hline$X_{2}$ & 0 & 0 & 0 & 0 & 0 \\
\hline$X_{3}$ & 0 & 0 & 0 & 0 & 0 \\
\hline$X_{4}$ & 2.2069 & 2.1559 & 1.9302 & 1.7342 & 1.6945 \\
\hline $\operatorname{Max} Z=$ & 7.6467 & 7.4936 & 7.1566 & 7.1026 & 7.0986 \\
\hline
\end{tabular}

\section{Conclusions}

In this study problem, it is assumed that randomly selected $a_{i j}(t=1, j=$ $1,2,8), b_{i}(i=2)$ model coefficients in the inequality constraints of the stochastic problem have Normal, ti Skew Normal and Skew-t distributions. Lingo 9.0 has been used to solve the deterministic equivalent problems of the stochastic problems. As a result; the solutions are compared to according to the decision variables and the objective function values of the deterministic problems. 
We compared the Normal distribution with Skew Normal distribution; $t$ distribution with Skew $t$ distribution to investigate the skewness effect; $t$ distribution with Normal distribution to investigate the tick tail effect; Skew- $t$ distribution with Skew Normal distribution to investigate the tick tail and skewness effect.

In Table 5 it is seen that for Normal distribution $(\lambda=0)$ the objective function has the highest value $z^{*}=7.68$, for the skewness effect we can say that; as the value of the skewness parameter $\lambda$. increases the value of the objective function decreases.

In Table 8 it is seen that for $t$ distribution $(\lambda=0)$ the objective function has the highest values $z^{*}=5.774,6.790,7.2932,7.6467$ for each $v=3,5,10,100$ degree of freedom values. As the value of the skevness parameter $\lambda$ increases the value of the objective function decreases. For two cases: Skew Normal and Skew- t distributions, we can see the skewness effect on the objective function values.

In Table 8 it is seen that; the objective function value for the Normal distribution $(\lambda=0)$ is $z^{*}=7.68$; the objective function values for the $t$ distribution $(\lambda=0, v=$ $3.5,10,100)$ is respectively $z^{*}=5.774,6.790,7.2932,7.6467$. For the tick tail effect, it can be said that that, for constant $\lambda=0$, the objective function has the closest value $\left(Z^{\prime}=7.6467\right)$ to normal distribution $\left(z^{*}=7.68\right)$ at $v=100$ degree of freedom value.

Finally, for both the skewness and the tick tail effects; when we compare the Skew Normal and Skew-t distributions; for constant $\lambda$ values, the objective function has the highest values at $v=100$. It can be said that for the tick tail effect; as the value of the $v$ increases the value of the objective function increases. For the large values of $v$, the objective function values of the models having Skew Normal and Skew-t distributions are closed to each other.

As a result, under the normality assumption; the CCSP problems can be solved with the different expected and the variance values. The solutions can be compared and $\mu, \sigma^{2}$ parameters effects can be invertigated. By the Skew Distributions are considered to be an extension of normal distribution; the skewness parameter and the tick tailness are included in the problem. So it can be seen the skewness and the tick tail effects. The Distributions was compared by the solutions. The Skew Distributions are very useful in the practise. They are the advantages in the CCSP.

Declaration of Competing Interest: The authors declare that they have no competing interest.

Acknowledgement The author would like to thank the Prof. Dr. Olcay Arslan and the referees for their many helpful comments and suggestions. 


\section{REFERENCES}

[1] Azzalini, A., A class of distributions which includes the normal ones, Scandinavian Journal of Statistics, 12 (1985), 171-178.

[2] Azzalini, A., Further results on a class of distributions which includes the normal ones, Statistica, 46 (2) (1986), 199-208.

[3] Azzalini, A. and Capitanio, A., Distributions generated by perturbation of symmetry with emphasis on a multivariate skew t distribution, Journal of the Royal Statistical Society, Series B ( Statistical Methodology), 65 (2) (2003), 367-389.

[4] Azzalini, A., The skew-normal distribution and related multivariate families (with discussion), Scandinavian Journal of Statistics, 32 (2005), 159-188.

[5] Arslan, O., A review on the univariate skew t-distributions, Far EAST Journal of Theoretical Statistics, 34(1) (2011), 17-34.

[6] Atalay, K. and Apaydin, A., Gamma distribution approach in change-constrained stochastic programming model, Journal of Inequalities and Applications, 1 (108) (2011), 3-13.

[7] Azzalini, A., The Skew Normal and Related Families, Cambridge University Press, (2014).

[8] Charnes, A. and Cooper, W.W., Chance constrained programming, Management Science, 6 (1959), 73- 79.

[9] Gupta, A.K., Chang, F.C., Huang, W.C., Some skew symmetric models, Random Operators and Stochastic Equations, 10 (2) (2002), 133-140.

[10] Gupta, A.K., Multivariate skew t distributions, Statistics: A Journal of Theoretical and Applied Statistics, 37 (4) (2003), 359-363.

[11] Hulsurkar et al., Fuzzy programming approach to multi-objective stochastic linear programming problems, Fuzzy Sets and Systems, 88 (1997), 173-181.

[12] Stancu-Minasian, I.M. and Wets M.J., A research bibliography in stochastic programming, Operational Research, 24 (6) (1976), 955-1975.

[13] Lin, T.I., Lee, J.C., Yen, S.Y., Finite mixture modelling using the skew normal distribution, Statistica Sinica, 17 (3) (2007), 909-927.

[14] O'Hagan, A. and Leonard, T., Bayes estimation subject to uncertainty about parameter constraints, Biometrika, 63 (1) (1976), 201-203.

[15] Yllmaz, M., Edgeworth series approximation for chi-square type chance constraints, Communications Faculty of Sciences University of Ankara Series A1, 56(2) (2007), 27-37.

[16] Yilmaz, M., Edgeworth series approximation for gamma type chance constraints, Selcuk Journal of Applied Mathematics, 10(1) (2009), 75-89.

[17] Yılmaz, M. and Potas, N., Solutions to change-constrained programming problems with exponential random variables by edgeworth approximation, Chaos Complexity Leadership Springer Proceedings in Complexity, (2016), 1-22. 